\title{
THE FERMENT-ANTIFERMENT BALANCE AND ITS RELATION TO THERAPEUSIS *
}

\author{
WILLIAM F. PETERSEN, M.D. \\ CHICAGO
}

For many years able and patient investigators have been occupied with the elaboration of the science of immunology. Numerous have been the achievements that have rewarded the direction of the foremost medical workers into these channels of thought and research; indeed, so splendid and satisfying have been many of them that we have gradually come to assume that therapeutic advance can take place only along these well demarcated lines. And yet, certain of the commoner infections have failed to yield to the intricate structure that has been reared in the effort to overcome them; failure which we have felt was due not to the method of attack, but to present limitations in the application of the theory by individual investigators. The study of tuberculosis illustrates the point most clearly. The end-result of innumerable immunologic studies has here left the problem of therapeusis practically unaltered; tuberculin therapy, introduced on a background of strictly immunologic research, while still offering the most favorable therapeutic result in selected cases, is no longer to be considered an immunizing agent in the specific sense of the term; the present time finds the laboratories devoted to tuberculosis research turning largely to studies along chemotherapeutic lines. It would seem justifiable, therefore, to explore the possibility of new avenues of approach to therapeutic problems; to determine whether or not some other broad basis can be found that, with proper study, will lead to fruitful therapeutic results. With this idea in mind the following outline is presented, not to be accepted as a completed study, but merely to offer briefly the rudiments of the problems when examined from a nonimmunologic standpoint.

The fundamental factor in overcoming bacterial intoxication not due to the soluble exotoxins lies in the ability of the cells or fluids of the invaded organism to digest the toxic protein fragments to their lowest degradation products and in this way overcome their deleterious effect. Digestion, too, no matter under what immunologic term we wish to classify the particular phase, must be considered the basis of the phenomena which have to do with overcoming bacterial invasion

* Submitted for publication July 25, 1917.

* From the Medical Clinic of Joseph L. Miller at the Cook County Hospital, and the Laboratory of Physiological Chemistry, College of Medicine, University of Illinois. 
itself. Might it not be warranted, therefore, to study in greater detail these digestive forces and their relation to pathologic conditions, in the hope of eventually being able to control ferment activity and achieve therapeutic results thereby?

To Abderhalden and his school in Germany we owe the beginning of such a movement; unfortunately, the center of interest was nearly shifted from a study of fundamental phenomena to particularistic and technical details involved in the clinical popularization of the so-called Abderhalden test for pregnancy. Abderhalden was undoubtedly prejudiced in favor of the idea of the specificity of proteolytic ferments because of the beautiful specificity displayed by the ferments that hydrolize the carbohydrates. When, however, one considers that the variety of carbohydrates with which ferments have to deal is relatively limited as contrasted with the practically endless combinations possible in the protein molecule, this prejudice is not necessarily logical. Possibly Abderhalden was influenced, too, by the immunologic conception of specificity; indeed, did hope to answer just this problem on the basis of highly specific proteases. The final demonstration that the Abderhalden reaction for pregnancy had not the merit of specificity emphasized by Abderhalden, unfortunately had a tendency to check further investigation in this field. Jobling was the first in this country to recognize the importance of, and to systematically study, ferment action in relation to pathologic conditions, and began his work some time previous to the investigations of Abderhalden in this particular line. In a series of some thirty odd articles, ${ }^{1}$ a broad experimental foundation has been laid on which it seems possible to attempt clinical investigation along lines not previously considered. In order to correlate the ideas which underlie the following studies it may be advisable to discuss briefly in this article some of the phenomena which Jobling has studied.

Vaughn, Matthes and Krehl, Friedberger and numerous other investigators have established the relation between intoxication and fever and the causal relation of the protein split products thereto. We are therefore justified in assuming that with bacterial invasion the intoxication of the organism is due to bacterial proteins; there is also a possibility that the proteins of the host themselves may, under certain conditions, give rise to toxic split products; the source, however, need not for the moment concern us.

If we are justified in ascribing major importance to these protein derivatives, we are equally justified in studying the mechanism of detoxication, which must involve the proteolytic ferments that will break down the toxic complexes to nontoxic forms. Several proteolytic ferments may occur in the serum: (1) The leucoproteases: (a)

1. Jour. Exper. Med., 1912-1916, 16-23. 
an alkaline active ferment capable of splitting native protein largely to the proteose stage; $(b)$ an acid active ferment with a similar range of activity; (c) an ereptase, active in both acid and alkaline reaction and splitting partially hydrolyzed proteins to the amino-acid stage. These ferments are derived from disintegrating but not from living leukocytes, and the fluctuations in the leukocyte count have therefore no direct bearing on the amount of these ferments in the serum. Of the ferments of this possible derivation only the ereptase is able to act in the presence of blood serum and tissue fluids, because the true proteases are inhibited by the antiferment. In a localized area, however, the amount of such ferments liberated may be sufficient to saturate the antiferment, and in this case digestion can go the entire stage from native protein to amino-acid. (2) Serum protease: a polyvalent, trypsin-like ferment, active in neutral, or even in slightly acid or alkaline reactions, is completely inhibited by the antiferment. It becomes active in the body only when the antiferment is removed or diminished, and is able to digest any native protein to the amino-acid stage. It is present in small amounts in normal human serum, but in relatively large amounts in certain of the lower animals. (3) Serum peptidase: a polyvalent ferment active in the presence of the serum antiferment, able to digest partly hydrolyzed proteins to the amino-acid stage and active in reactions similar to those favorable for protease action. It is present in normal human serum in small amounts.

These ferments should not be confused with the alexin, or complement, which is slightly less resistant to heat and is destroyed by the lipoid solvents. Whether or not these ferments always act in a lytic capacity, or may under certain conditions become synthetic, is a question as yet unsettled.

Inasmuch as the first two types of ferment are active only under special conditions and are not present in more than small amounts in the serum at any time, they are of relatively less import than the last ferment, the peptidase. It is at once apparent that we are dealing here with the detoxicating agent, a ferment that hydrolyzes proteins from the toxic stages - albumoses (proteoses) and peptones - to the nontoxic amino-acids. A mobilization of this ferment could then be considered only as of beneficial significance, never as a factor in the production of an intoxication, and, since it is active in the presence of the antiferment, the fluctuations of the latter in titer would have no effect on the detoxicating force. An increase in the amount of this ferment occurring spontaneously during the course of disease should, therefore, be coincident with clinical improvement and, conversely, the disappearance of the ferment should permit the accumulation of toxic split products and an increase in intoxication. An increase induced arti- 
ficially, if possible, should be equally effective as a measure of detoxication.

When we turn to the true proteases we deal with a reaction of which the possibilities are more complex for two principal reasons: (a) the ferment action may involve the splitting of native proteins to the higher split products and may conceivably, at some time in the process, give rise to intoxication; $(b)$ the ferment action is balanced by the presence of the antiferment, and we have therefore to deal with two varjable factors. For simplicity we may take two concrete examples. Suppose that a pneumonic focus with its mass of cellular detritus and fibrin represents, for the normal tissue, simply so much foreign material from which it must free itself by digestion; as long as the autolysis of this foreign mass proceeds slowly, higher and toxic split products will be absorbed as such; digestion proceeds slowly, because the leukocytes are still living, and therefore not "shedding" their ferment as they should, and because of the great increase in the antiferment of the serum and tissue fluids which takes place early in the infection. According to the hypothesis presented, if the antiferment titer is now lowered and at the same time sufficient protease and peptidase are liberated, we might expect a temporary increase in the degree of intoxication (because of the rapid absorption of toxic split products) followed by a detoxication when autolysis is actively initiated. A therapeutic ideal might therefore be sought in just such an alteration of the ferment-antiferment balance.

Let us assume, on the other hand, that we are dealing with a quiescent tubercle with just sufficient encapsulation by scar tissue to prevent any excretion of toxic material from the focus. If, for any reason, the serum alteration above mentioned should occur in such a patient, we might expect a considerable degree of intoxication due to the digestion of the protective connective tissue and of the central necrotic mass, together with the liberation of accumulated toxic material preformed in the necrotic focus. Theoretically, it might seem warranted to endeavor to increase the antiferment in this condition in order to check protease activity and protect the tubercle from digestion. In a subsequent article this reaction and its relation to our present empirical therapy will be discussed in detail. It will be evident from the two examples outlined that fluctuations in the digestive balance of the tissues and serum may effect certain pathologic processes in a most fundamental way.

When considering the factors in the balance, it is apparent that we are compelled to consider the antiferment, or antitrypsin, as it is commonly designated, as an integral part of the mechanism under consideration. Here we enter the field of lipoid bodies. The antiferment is not an antibody in the immunologic sense, although it was early so 
considered; it consists of the highly dispersed unsaturated lipoids of the serum and lymph and its titer varies, therefore, with at least three conditions: ( $a$ ) the amount of lipoids present; $(b)$ the dispersion, and (c) the chemical structure; that is, the degree of unsaturation; all conditions that are subject to considerable variation and any one of which may account for wide fluctuations in the titer commonly observed. Thus, lessening the dispersion by acidifying, by salting and by heating to a sufficient degree, inactivates the antiferment; physical adsorption by certain chemically inert adsorbing surfaces lowers the titer; solution of the lipoids in chloroform and ether removes the antiferment from the serum. The most comparable substances, in their antitryptic property, which we have available, are the soaps of the unsaturated fatty acids. There is considerable ground for the belief that the antiferment lipoids are in more or less intimate physical combination with the serum albumin with which fraction they are thrown down on the usual methods of separation of the serum proteins.

It is known that the antiferment is greatly augmented during certain diseases, notably in the acute infections, in pregnancy, in carcinoma and cachectic states in general; following anaphylactic and other protein shock, and in certain pathologic processes in the central nervous system of a degenerative character; in other words, it forms part of a rather general "reaction" phenomenon. This seems purposeful, in that an increase in the antiferment power would tend to counteract the heightened destruction of the proteins of the body commonly observed in toxic conditions. The antiferment of the lymph stream is appreciably increased after feeding, although the increase in the blood stream is only moderate in amount; in starvation, on the other hand, the antiferment shows a progressive decrease.

We deal, then, with the probability that the antiferment may be augmented by selective diet, by shock therapy (including vaccines), and possibly by the intravenous injection of the proper lipoids. Another factor enters, however, into the regulatory mechanism, namely, the fact that the amount of lipoids in the serum is related to the lipolytic activity developed therein. That is, when the lipolytic activity is low, as for instance, in pregnancy, in carcinoma, in the acute infections and in advancing tuberculosis, an accumulation of lipoids will take place in the serum and, as a corollary, the antiferment titer will increase. Conversely, a strengthening of the lipolytic activity will metabolize the serum lipoids more rapidly and in this way decrease the antiferment titer. It is apparent, therefore, that the regulation of the antiferment titer is dependent on several factors, some of which may be amenable to artificial and therapeutic control.

Empirically, we have probably been doing precisely this in our use of iodin and the iodids. These are used therapeutically in a great 
number of disease conditions, but chiefly, and with universal acceptance, to hasten the resolution of necrotic tissues, such as the gummas of late syphilis. It has been demonstrated experimentally that the iodids will increase the rate of autolysis to a considerable degree. Inasmuch as the effect is not found to be directly on the ferment, we must seek to find some other explanation. In this connection it was noticed in vitro experiments that the iodids lessened the antiferment titer of the serum, and later experiments carried out in clinical cases revealed that a lowering of the antiferment titer took place progressively under iodid therapy. We have then, it seems, one of the means of influencing the ferment-antiferment balance in common use.

The direct intravenous injection of ferments has not yet been used with success, because of the toxicity of the perparations employed. Hiss and Zinsser, in their interesting work commenced a number of years ago, employed leukocytic extract subcutaneously with encouraging results, although the possibility is not excluded, as Zinsser and Tsen point out in a recent article, that the results achieved are due to a general shock reaction rather than to the absorption of ferments. The direct increase of ferments is at least one method of approach worthy of further investigation.

If protein splitting with its resulting formation of toxic split products is at the basis of many of the intoxications with which we have commonly to deal in acute and chronic conditions, then the detoxication by means of ereptases and similar ferments should be of considerable importance. From experimental observations it seems probable, at any rate, that by means of alterations in the ferment-antiferment balance we may be able to attack certain therapeutic problems from this relatively simple point of view. 